\title{
SIMULATION OF A FLOW THROUGH A RIGID POROUS MEDIUM SUBJECTED TO A CONSTRAINT
}

\author{
R. M. Saldanha da Gama ${ }^{a}$, \\ J. J. Pedrosa Filho ${ }^{a}$, \\ and M. L. Martins-Costa ${ }^{b}$ \\ ${ }^{\text {a}}$ Universidade do Estado do Rio de Janeiro \\ Departamento de Engenharia Mecânica FEN \\ Rua São Francisco Xavier, 524, CEP 20550-013, \\ Rio de Janeiro, RJ, Brasil \\ ABSTRACT \\ This work uses a previuosly proposed mathematical model to study the \\ filling up of an unsaturated rigid porous medium by a liquid identifying \\ the transition from unsaturated to saturated flow. This model accounts \\ for the physical upper bound of the fluid fraction that depends on the \\ volume of the pores and employs a mixture theory to describe the flow. \\ The mathematical description of the phenomenon leads to a nonlinear \\ hyperbolic system. In order to solve this system, the space of admissible \\ solutions must be enlarged to admit discontinuous solutions that may be \\ shock waves. The complete solution of a Riemann problem associated to \\ the system of conservation laws satisfying the constraint given by the \\ rsggama@gmail.com, zejulio@terra.com.br \\ saturation upper bound is presented. Some meaningful results are \\ ${ }^{\mathrm{b}}$ Universidade Federal Fluminense \\ Departamento de Engenharia Mecânica \\ TEM/PGMEC \\ Laboratório de Mecânica Teórica e Aplicada \\ Rua Passo da Pátria, 156, CEP 24210-240, \\ Niterói, RJ, Brasil \\ Received: October 25, 2013 \\ Revised: November 27, 2013 \\ Keywords: Flow through unsaturated porous media, transition \\ unsaturated/saturated flow, constrained unknown, Riemann problem, \\ Accepted: December 30, 2013 \\ shock waves.
}

\section{NOMENCLATURE}

$\mathbf{b}_{F} \quad$ body force per unit mass, $\mathrm{m} / \mathrm{s}^{2}$

c constant

$\mathrm{L} \quad$ porous slab thickness, $\mathrm{m}$

$\mathbf{m}_{F} \quad$ momentum supply on fluid constituent, $\mathrm{N} / \mathrm{m}$

$p \quad$ pressure, $\mathrm{N} / \mathrm{m}^{2}$

$p$ dimensionless pressure

$t$ time, $\mathrm{s}$

$\mathbf{T}_{F} \quad$ partial stress tensor, $\mathrm{N} / \mathrm{m}^{2}$

$u \quad$ dimensionless fluid constituent velocity on $x$ direction

$\mathbf{v}_{F} \quad$ fluid constituent velocity in the mixture, $\mathrm{m} / \mathrm{s}$

$v \quad$ fluid constituent velocity on $x$ direction, $\mathrm{m} / \mathrm{s}$

$x \quad$ Cartesian coordinate, $\mathrm{m}$

\section{Greek symbols}

$\varepsilon \quad$ porous matrix porosity

$\theta$ dimensionless time

$\rho_{F} \quad$ fluid constituent mass density $\mathrm{kg} / \mathrm{m}^{3}$

$\rho_{f} \quad$ fluid mass density $\mathrm{kg} / \mathrm{m}^{3}$

$\varphi \quad$ fluid fraction

$\psi \quad$ saturation

\section{Subscripts}

$L \quad$ left state

$R \quad$ right state

* $\quad$ intermediate state

$0 \quad$ initial state for fluid fraction

\section{INTRODUCTION}

This work employs a physically realistic mathematical model to represent the filling up of an unsaturated rigid porous matrix by a fluid, identifying the transition from unsaturated to saturated flow, by imposing a constraint (an upper bound) on the saturation proposed by Saldanha da Gama et al. (2012). According to a comprehensive review by Alazmi and Vafai (2000), an adequate description of this transition remains an open subject. The mechanical modeling uses a mixture theory approach to model a porous medium bounded by an impermeable wall.

Distinct physical applications may give rise to constrained hyperbolic systems. Among them twophase flows of gas and liquid models, compressible plasticity with shocks and models for traffic of vehicles or crowds These may be described with the help of fluid dynamic models with distinct degrees of complexity that may account for accidents on roads, traffic jams or toll gates. Bouchut et al. (2000) model 
the dynamics of gas occlusions on pipes by studying two-phase flows of gas and liquid models, for incompressible liquids, ranging from nonconservative conservation laws with relaxation to a system of pressureless gases with concentration constraint and undetermined pressure. Després et al. (2011) present a mathematical framework for constrained weak solutions of hyperbolic equations, to model compressible plasticity with shocks. This developed weak formalism allows accounting for both Tresca and Von Mises plasticity criteria.

Concerning traffic flow models, Daganzo (1995) observed that models like the Euler equations for gas dynamics may lead to absurd behavior like vehicles going backwards. The Aw-Rascle model, proposed by Aw and Rascle (2000), ensures that both density and velocity remain nonnegative and correcting the absurd behavior detected by Daganzo (1995). Berthelin et al. (2008) studied the dynamics of traffic jams by proposing a traffic flow model consisting of a pressureless gas dynamics system under a maximal constraint on the density - so that the density constraint is always preserved. Aiming at predicting traffic accidents, Herty and Schleper (2011), consider different mathematical models that can describe macroscopically the traffic flow, accounting for the mathematical properties of a coupled macroscopic second-order traffic model with different pressure laws on the connected roads. Colombo and Goatin (2007) consider a single hyperbolic equation (Cauchy problem) subjected to a local variable unilateral constraint on the flux in order to model a tollgate along a highway. The total number of vehicles is conserved and the traffic speed is assumed to be a function of traffic density. This work could be compared with the model proposed by Saldanha da Gama et al. (2012) in which a system of two partial differential equations is considered. However, Colombo and Goatin (2007) consider a variable unilateral constraint only on the boundary, while Saldanha da Gama et al. (2012) consider a constraint in the whole domain for all time.

In order to avoid solutions without physical meaning, Saldanha da Gama (1986) proposed a constitutive relation for the partial pressure accounting for a geometrical bound that results from the fluid incompressibility and the porous medium rigidity. Later, an improvement to this constitutive relation was proposed by Martins-Costa and Saldanha da Gama (2011) in which, the unilateral geometrical constraint for the fluid fraction, instead of being assumed in the whole domain, is considered only in a convenient neighborhood of the porosity (provided that the fluid fraction is smaller than the porosity). Since Martins-Costa and Saldanha da Gama (2011) assured continuity for the pressure and for its first derivative, the Riemann invariants associated to the problem could be analytically computed.

It is important to note that while in Saldanha da Gama et al. (2012) the porous medium can actually be saturated by the fluid, the equation proposed by Saldanha da Gama (1986) only imposes a physical behavior for the fluid preventing it to saturate the porous matrix.

\section{MECHANICAL MODEL}

The unsaturated porous medium is modeled using a mixture theory approach (Atkin and Craine, 1976; Rajagopal and Tao, 1995) in which three overlapping continuous constituents are considered: a solid (a rigid, homogeneous and isotropic porous matrix), a liquid (an incompressible fluid) and an inert gas, assumed with very low mass density; which was included to account for the compressibility of the system as a whole. Since the solid constituent is rigid and the gas constituent is inert it suffices to solve mass and momentum equations for the liquid (fluid) constituent, which are given by:

$$
\begin{gathered}
\frac{\partial \rho_{F}}{\partial t}+\nabla \cdot\left(\rho_{F} \mathbf{v}_{F}\right)=0 \\
\rho_{F}\left[\frac{\partial \mathbf{v}_{F}}{\partial t}+\left(\nabla \mathbf{v}_{F}\right) \mathbf{v}_{F}\right]=\nabla \cdot \mathbf{T}_{F}+\mathbf{m}_{F}+\rho_{F} \mathbf{b}_{F}
\end{gathered}
$$

where $\rho_{F}$, the fluid constituent mass density, represents the local ratio between the fluid constituent mass and the corresponding volume of mixture, $\mathbf{m}_{F}$ is a momentum supply acting on the fluid constituent due to its interaction with the remaining constituents of the mixture and the partial stress tensor $\mathbf{T}_{F}$ is analogous to Cauchy stress tensor in Continuum Mechanics. These latter quantities require constitutive assumptions. The ratio between the fluid fraction $\varphi$ and the porous matrix porosity $\varepsilon$ is defined as the saturation $\psi$, so that

$$
\psi=\frac{\varphi}{\varepsilon}=\frac{\rho_{F}}{\varepsilon \rho_{f}} \quad 0<\psi \leq 1 \quad \text { everywhere }
$$

where $\rho_{f}$ is the actual mass density of the fluid (in a Continuum Mechanics viewpoint). Constitutive relations are now presented for the partial stress tensor considering the normal fluid stresses dominant over shear stresses and interphase tractions as proposed by Allen (see Saldanha da Gama et al., 2012 and references therein) and for the momentum source $\mathbf{m}_{F}$ as given by a term related to the fluid constituent velocity, usually called Darcian and term a term related to the saturation gradient. (See Saldanha da Gama et al., 2012 and references therein.)

$$
\begin{aligned}
& \mathbf{m}_{F}=-\frac{\mu_{f}}{K} \varphi^{2} \mathbf{v}_{F}-\frac{\mu_{f} D}{K} \nabla \varphi \\
& \mathbf{T}_{F}=-\varphi \bar{p} \mathbf{I}
\end{aligned}
$$


where $\mu_{f}$ is the fluid viscosity, $K$ the porous matrix specific permeability (in a Continuum Mechanics viewpoint), $D$ a diffusion coefficient and $\bar{p}$ is a pressure (assumed constant while the flow is unsaturated). The Darcian term (first term of the momentum source) is neglected, since unsaturated flows through porous media are characterized by a strong dependence of the motion on the saturation.

At this point the mechanical model can be built by combining the balance equations (1) with the constitutive relations (3) together with the assumption that the pressure $p=\hat{p}(\varphi)$ may be redefined as $p=\bar{p} \varphi+\left(\mu_{f} D / K\right) \varphi$. Also, assuming absence of body forces, that all quantities depend on the time $t$ and on the position $x$ only, and that $v$ is the only nonvanishing component of the fluid constituent velocity $\mathbf{v}_{F}$, the problem may be written as

$$
\begin{gathered}
\frac{\partial \phi}{\partial t}+\frac{\partial}{\partial x}(\varphi v)=0 \\
\frac{\partial}{\partial t}(\varphi v)+\frac{\partial}{\partial x}\left(p+\varphi v^{2}\right)=0
\end{gathered}
$$

The unknowns $\varphi$ and $v$, depending on position $x$ and on time $t$, represent, respectively, the fluid fraction and the fluid constituent velocity in the description of the flow through an unsaturated porous medium. Physically, there is no restriction on the velocity, which can assume any real value, but the fluid fraction $\varphi$ must be positive valued and smaller than (or equal to) the porosity $\varepsilon$, otherwise the problem is no longer physically consistent. Since the maximum amount of fluid is bounded by the porous medium porosity, the fluid fraction is a constrained unknown given by

$0<\varphi \leq \varepsilon, \quad \forall x, \forall t>0$

In addition, in this work, the following relationship (Saldanha da Gama et al., 2012) is considered

$$
p=c^{2} \varphi, \quad 0<\varphi \leq \varepsilon
$$

It is important to note that the following relations must hold for a rigid and homogeneous porous medium: $p=\hat{p}(\varphi)$ for $0<\varphi<\varepsilon$, characterizing unsaturated flow and $\hat{p}(\varepsilon) \leq p<\infty$ for $\varphi=\varepsilon$, characterizing saturated flow (Saldanha da Gama et al., 2012).

The governing equations may be rewritten in a dimensionless form as follows

$$
\begin{aligned}
& \frac{\partial \varphi}{\partial \theta}+\frac{\partial}{\partial x}(\varphi u)=0 \\
& \frac{\partial}{\partial \theta}(\varphi u)+\frac{\partial}{\partial x}\left(p_{\square}+\varphi u^{2}\right)=0 \\
& \varphi \leq \varepsilon
\end{aligned}
$$

in which

$$
\theta=\frac{t}{c}, \quad u=\frac{v}{c} \quad \text { and } \quad p=\frac{p}{c^{2}}
$$

\section{PROBLEM FORMULATION AND SOLUTION}

The problem is defined in an infinite porous medium with constant porosity $\varepsilon$ and an initial constant fluid fraction $\varphi$ such that $0<\varphi<\varepsilon$. Supposing that the fluid is initially at rest and, suddenly, a motion takes place in such a way that

$$
\begin{aligned}
& u=u_{L}=\text { constant for } t=0,-\infty<x<0 \\
& u=u_{R}=\text { constant for } t=0, \quad 0<x<\infty
\end{aligned}
$$

This phenomenon is mathematically represented as follows

$$
\begin{aligned}
& \frac{\partial \varphi}{\partial \theta}+\frac{\partial}{\partial x}(\varphi u)=0 \\
& \frac{\partial}{\partial \theta}(\varphi u)+\frac{\partial}{\partial x}\left(p_{\square}+\varphi u^{2}\right)=0, \\
& \text { with } \quad p=\varphi \text { for } \varphi<\varepsilon \\
& \varphi \leq \varepsilon \quad \begin{array}{l}
\left(\varphi_{0}, u_{L}\right) \text { for } t=0,-\infty<x<0 \\
\left(\varphi_{0}, u_{R}\right) \text { for } t=0, \quad 0<x<\infty
\end{array}
\end{aligned}
$$

It is important to note that problem (10) is called a Riemann problem.

The solution (in a generalized sense) of this Riemann Problem is reached by connecting the left state and the right state to an intermediate state reached by means of continuous and/or discontinuous functional relationships. When the inequality

$$
u_{L}>u_{R}
$$

takes place, there is no continuous connection.

In addition, in such cases, when the inequality

$$
2\left(\sqrt{\frac{\varepsilon}{\varphi_{0}}}-\sqrt{\frac{\varphi_{0}}{\varepsilon}}\right)<u_{L}-u_{R}
$$


holds, the porous medium is saturated. In such cases, the intermediate fluid fraction becomes equal to the porosity, while $p$ is no longer given by the constitutive equation $p=\varphi$. In these cases, $p$ is obtained directly from the balance equations.

Denoting by $\left(\varphi_{*}, u_{*}\right)$ the intermediate state, it comes that the intermediate pressure is given by

$$
p_{*}=\varphi_{*}=\frac{\varphi_{0}}{4}\left\{\frac{u_{L}-u_{R}}{2}+\sqrt{\left(\frac{u_{L}-u_{R}}{2}\right)^{2}+4}\right\}^{2}
$$

provided $\varphi_{*}$ is less than $\varepsilon$. In these cases, the intermediate pressure $p$ is also given by $\varphi_{*}$. On the other hand, when the above equation gives rise to $\varphi_{*}$ greater than or equal to the porosity, in order that the problem remains physically consistent, $p_{* *}$, the intermediate pressure is given by

$$
p_{*}=\varphi_{0}+\frac{\left(\frac{u_{L}-u_{R}}{2}\right)^{2}}{\left(\frac{1}{\varphi_{0}}-\frac{1}{\varepsilon}\right)}
$$

while the intermediate velocity $u_{*}$ is given by

$$
u_{*}=\frac{u_{L}+u_{R}}{2}-\left(\sqrt{\frac{\varphi_{*}}{\varphi_{0}}}-\sqrt{\frac{\varphi_{0}}{\varphi_{*}}}\right) \sqrt{\left(\frac{p_{*}-\varphi_{0}}{\varphi_{*}-\varphi_{0}}\right)}
$$

The speeds of propagation can be computed from the jump conditions being given by

$$
\begin{aligned}
& S_{1}=\frac{\varphi_{0} u_{L}-\varphi_{*} u_{*}}{\varphi_{0}-\varphi_{*}} \quad \text { (speed of shock 1) } \\
& S_{2}=\frac{\varphi_{0} u_{R}-\varphi_{*} u_{*}}{\varphi_{0}-\varphi_{*}} \quad \text { (speed of shock 2) }
\end{aligned}
$$

At this point, assuming $u_{L}>u_{R}$, the solution of the Riemann problem represented by equation (10), in a generalized sense, may be represented by the flowing equations

$$
(\varphi, u)=\left\{\begin{array}{l}
\left(\varphi_{0}, u_{L}\right) \text { if }-\infty<\frac{x}{\theta}<\frac{\varphi_{0} u_{L}-\varphi_{*} u_{*}}{\varphi_{0}-\varphi_{*}} \\
\left(\varphi_{*}, u_{*}\right) \text { if } \frac{\varphi_{0} u_{L}-\varphi_{*} u_{*}}{\varphi_{0}-\varphi_{*}}<\frac{x}{\theta}<\frac{\varphi_{0} u_{R}-\varphi_{*} u_{*}}{\varphi_{0}-\varphi_{*}} \\
\left(\varphi_{0}, u_{R}\right) \text { if } \frac{\varphi_{0} u_{R}-\varphi_{*} u_{*}}{\varphi_{0}-\varphi_{*}}<\frac{x}{\theta}<\infty
\end{array}\right.
$$

Figure 1 shows the relationship between the ratio of dimensionless pressure and porosity $p_{* *} / \varepsilon$ and the difference of dimensionless velocities $u_{L}-u_{R}$ for two values of the ratio between the initial fluid fraction and the porosity $\varphi_{0} / \varepsilon$, characterizing two initial value problems. Two distinct sets of curves are represented in figure 1 showing two sets of curves. The upper lines represent the relationship between $p_{*} / \varepsilon$ and $u_{L}-u_{R}$ for $\varphi_{0} / \varepsilon=0.5$ while the lower ones were obtained for $\varphi_{0} / \varepsilon=0.3$. Also, the thicker lines correspond, for both considered cases to the lines that represent the behavior of the ratio $p_{*} / \varepsilon$ with the variation of the dimensionless velocity difference $u_{L}-u_{R}$.

The black dot indicates the transition from unsaturated to saturated flow. At the left side of each black dot, the inferior lines hold. At the right side, where the flow becomes saturated and the constraint must be imposed, the superior lines hold.

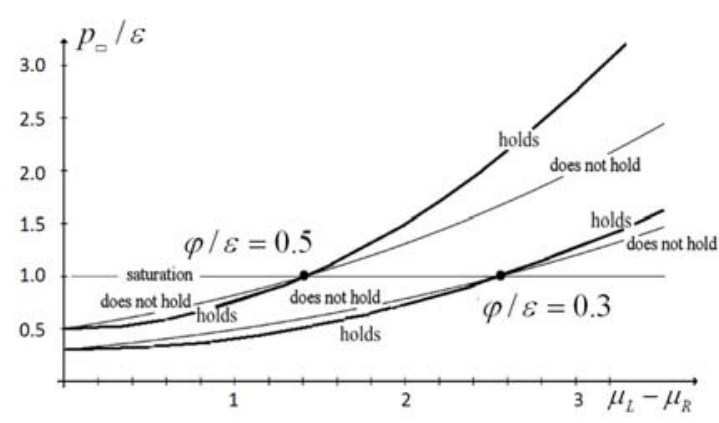

Figure 1. Relationship between the ratio $p_{* *} / \varepsilon$ and $u_{L}-u_{R}$ for two values of the ratio $\varphi_{0} / \varepsilon$.

It is important to note that whenever the constraint is not satisfied the results are no longer physically reasonable - which is indicated by "does not hold".

Figure 2 and 3 present the exact solution of the Riemann problem represented by equation (10), presented in equation (17).

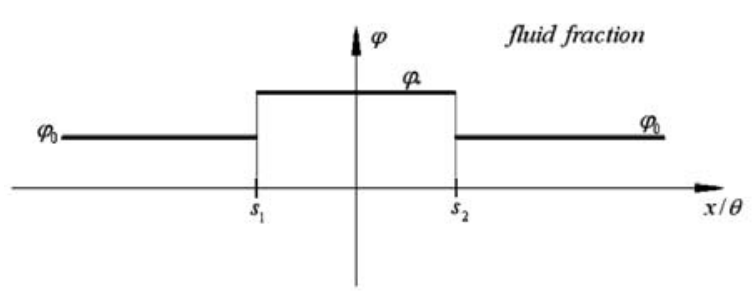

Figure 2. Solution of Riemann problem (9) - Fluid fraction as a function of the ratio $x / \theta$. 
Since $u_{L}>u_{R}$, the solution is shock $1 /$ shock 2 , and the speeds of propagation given by equation (15) are also depicted in figures 2 and 3. As indicated in Equation (17), the fluid fraction equals $\varphi_{0}$, except for the region $S_{1}<x / \theta<S_{2}$, in which it equals $\varphi_{*}$, as depicted in figure 2 . In figure 3 , the initial condition stated in equation (9) is presented, considering $u_{L}>u_{R}$, and the value for intermediate velocity $u_{*}$ is given by equation (15).

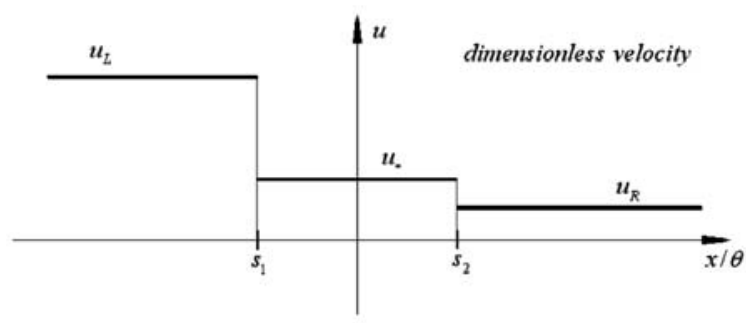

Figure 3. Solution of Riemann problem (9) Dimensionless velocity as a function of the ratio $x / \theta$.

\section{FINAL REMARKS}

This work presented an exact solution for flows through unsaturated porous media, identifying the transition from unsaturated to saturated flow. The mechanical model included a constraint that must be satisfied to build physically realistic generalized solutions, valid for any initial data.

The complete solution of a constrained nonlinear hyperbolic problem with shock waves - an associated Riemann problem containing a restriction (an upper bound for the fluid fraction, represented by the porosity), was presented as well as its application to flows through porous media.

\section{ACKNOWLEDGEMENTS}

The authors R. M. Saldanha da Gama and M. L. Martins-Costa gratefully acknowledge the financial support provided by the Brazilian agency CNPq.

\section{REFERENCES}

Alazmi, B., and Vafai, K, 2000, Analysis of Variants within the Porous Media Transport Models, Journal Heat Transfer, Vol. 122, pp. 303-326.

Atkin, R. J., and Craine, R. E., 1976, Continuum Theories of Mixtures. Basic Theory and Historical Development, Quarterly Journal of Mechanics and Applied Mathematics, Vol. 29, pp. 209-244

Aw, A., and Rascle, M., 2000, Resurrection of "Second Order" Models of Traffic Flow, SIAM
Journal on Applied Mathematics, Vol. 60, No. 3, pp. 916-938.

Berthelin, F., Degond, P., Delitala, M., and Rascle, M., 2008, A Model for the Formation and Evolution of Traffic Jams, Archive for Rational Mechanics and Analysis, Vol. 187, pp. 185-220.

Bouchut, F., Brenier, Y., Cortes, J. and Ripoll, J. F., 2000, A Hierarchy of Models for Two-Phase Flows, Journal Nonlinear Science, Vol. 10, pp. 639660.

Colombo, R. M. and Goatin, P., 2007, A Well Posed Conservation Law with a Variable Unilateral Constraint, J. Differential Equations, Vol. 234, pp. 654-675.

Daganzo, C., 1995, Requiem for Second Order Fluid Approximations of Traffic Flow, Transportation Research Part B: Methodological, Vol. 29B, pp. 277-286.

Herty, M., and Schleper, V., 2011, Traffic Flow with Unobservant Drivers, Journal of Applied Mathematics and Mechanics, Vol. 91, No. 10, pp. 763-776.

Martins-Costa, M. L. and Saldanha da Gama, R. M., 2011, A New Constitutive Equation for Unsaturated Flows of Incompressible Liquids through Rigid Porous Media, Journal Porous Media, Vol. 14, pp. 205-217.

Rajagopal, K. R., and Tao, L., 1995, Mechanics of Mixtures, Series Advances in Math. Appl. Sci., Vol. 35, World Scientific, Singapore

Saldanha da Gama, R. M., 1986, A New Mathematical Modelling for Describing the Unsaturated Flow of an Incompressible Liquid through a Rigid Porous Medium, International Journal of Non-Linear Mechanics, Vol. 40, pp. 5968.

Saldanha da Gama, R. M., Pedrosa Filho, J. J., and Martins-Costa, M. L., 2012, Modeling the Saturation Process of Flows through Rigid Porous Media by the Solution of a Nonlinear Hyperbolic System with One Constrained Unknown, Journal of Applied Mathematics and Mechanics, Vol. 92, No. 11-12, pp. 921-936. 